\title{
The early evolution of Globular Clusters: the case of NGC 2808
}

\author{
Francesca D'Antona ${ }^{1}$ and Vittoria Caloi ${ }^{2}$ \\ ${ }^{1}$ INAF - Osservatorio Astronomico di Roma, via Frascati 33, 00127 Roma, Italy; \\ dantona@mporzio.astro.it \\ ${ }^{2}$ Istituto di Astrofisica Spaziale e Fisica Cosmica, C.N.R., Via Fosso del Cavaliere, I-00133 Roma, \\ Italy; caloi@rm.iasf.cnr.it
}

\begin{abstract}
Enhancement and spread of helium among globular cluster stars have been recently suggested as a way to explain the horizontal branch blue tails, in those clusters which show a primordial spread in the abundances of $\mathrm{CNO}$ and other elements involved in advanced $\mathrm{CNO}$ burning. Helium enhancement is unavoidable, if the matter responsible for the abundance spreads is identified with the matter lost by massive asymptotic giant branch stars, which evolve during the early phases of globular cluster life (D'Antona et al. 2002).

In this paper we examine the implications of the hypothesis that, in many globular clusters, stars were born in two separate events: an initial burst (first generation), which gives origin to probably all high and intermediate mass stars and to a fraction of the cluster stars observed today, and a second, prolonged star formation phase (second generation) in which stars form directly from the ejecta of the intermediate mass stars of the first generation. In particular, we consider in detail the morphology of the horizontal branch in NGC 2808 and argue that it unveils the early cluster evolution, from the birth of the first star generation to the end of the second phase of star formation. This framework provides a feasible interpretation for the still unexplained dichotomy of NGC 2808 horizontal branch, attributing the lack of stars in the RR Lyr region to the gap in the helium content between the red clump, whose stars are considered to belong to the first stellar generation and have primordial helium, and the blue side of the horizontal branch, whose minimum helium content reflects the helium abundance in the smallest mass $\left(\sim 4 \mathrm{M}_{\odot}\right)$ contributing to the second stellar generation. This scenario provides constraints on the required Initial Mass Function, in a way that a great deal of remnant neutron stars and stellar mass black holes might have been produced.
\end{abstract}

Subject headings: globular clusters: general — globular clusters: individual NGC 2808 stars: horizontal branch — neutron stars — millisecond pulsars — black holes

\section{Introduction}

Chemical inhomogeneities in globular cluster (GC) stars are giving crucial clues on the formation and evolution of this most ancient stellar population. Self- enrichment mechanisms are being examined as ones of the possible - and most plausible - causes of abundance spread in many GCs.

The question of self-enrichment in GCs was raised for the first time in connection with the wide spread in metallicity observed in $\omega$ Cen (Freeman \& Rodgers 1975; Freeman \& Norris 1981; Mallia \& Pagel 
1981). Recently there has been a large amount of work on this problem (f.e., Smith et al. 2000), an aspect of which is the ability of a cluster to retain the matter lost by asymptotic giant branch (AGB) stars. Gnedin et al. (2002) observe that $\omega$ Cen is not special among GCs in this respect, and that a few dozen other clusters should be capable of doing so. In fact, contrary to a substantial uniformity of abundance of heavy elements (apart from the quoted $\omega$ Cen), light elements that are susceptible to abundance changes from proton-capture reactions, such as the pp, CN, ON, NeNa, and MgAl cycles, exhibit star-to-star abundance variations in many GCs, far in excess of the modest variations seen in halo field stars -see, e.g., Smith (1987), Kraft (1994), and Sneden (1999,2000). In recent years, observations of these abundance spreads at the turnoff and among the subgiant stars (e.g., Gratton et al. 2001) have shown that these anomalies must be attributed indeed to some process of self-enrichment occurring at the first stages of the life of the cluster, starting as soon as all the supernovae have already exploded (expelling from the clusters their high velocity ejecta) and the massive AGB stars begin to evolve. At an epoch starting $\sim 510^{7} \mathrm{yr}$ from the birth of the first stellar generation, the massive AGBs lose mass through low velocity winds, so that it can be reasonably speculated that these winds remain inside the cluster.

The massive AGB envelopes are the ideal place to manifacture elements through nuclear reactions in which proton captures are involved, as they are subject to hot bottom burning (HBB) (e.g. Ventura et al. 2001; Ventura, D'Antona, \& Mazzitelli 2002), although a quantitative reproduction of the observed abundance spreads, e.g., of the oxygen vs. sodium anticorrelation (Denissenkov \& Herwig 2003; Ventura et al. 2004), is still far from being available. One important feature of this hypothesis is that the chemistry of the stars which are formed in the second generation is not random, but reflects the continuous variation of HBB conditions with the decrease of the progenitor evolving mass, until the star formation process ends (see the discussion in D'Antona 2003). Thus the ejecta of these stars can be the source of the star-to-star abundance variations.

The hypothesis that the ejecta are accreted on the already formed stars (D'Antona, Gratton, \& Chieffi 1983; Thoul et al. 2002) has been recently falsified by several observations -see, for a summary of these problems, Gratton, Sneden \& Carretta (2004). In this paper we further explore the hypothesis that such ejecta continuously form second generation stars for a time lasting about 200Myr (D'Antona et al. 2002).

We argue that NGC 2808 is one of the clusters able to retain the AGB ejecta, even if to a degree lower than wCen, which is capable of retaining at least part of SNII ejecta (f.e., Smith et al. 2000). Large variations in $\mathrm{Na}$ abundance have been observed among the stars of this cluster (Carretta et al. 2003), at all luminosity levels along the red giant $(\mathrm{RG})$ branch. So members of this cluster have suffered pollution by $\mathrm{Na}$-enriched matter, as observed in M13, M5, M4, M92, M15, NGC 6752. We do not have information on main sequence abundances, as in NGC 6752 (Gratton et al. 2001) where a primordial origin for chemical inhomogeneities appears the only possible explanation, nevertheless the hypothesis of self- enrichment in NGC 2808 is supported by the observed $\mathrm{Na}$ - spread. We expect the most massive and concentrated clusters to be in the best conditions to retain mass lost by cluster members. NGC 2808 has a central density of $\log (\rho)=4.9\left(\mathrm{M}_{\odot} / \mathrm{pc}^{3}\right.$, Pryor \& Meylan 1993), among the largest densities found in Galactic GCs; the total visual magnitude is $\mathrm{M}_{\mathrm{v}}=-9.26$ (Harris 1996), which, assuming a ratio $M / \mathrm{L}_{\mathrm{v}}=3$, gives a total cluster mass well above $10^{6} \mathrm{M}_{\odot}$. According to Gnedin et al. (2002, Fig. 2), NGC 2808 would be at present quite able of retaining stellar winds of about $15 \mathrm{~km} \mathrm{~s}^{-1}$, the characteristic terminal velocity of AGB winds (Loup et al. 1993). Since according to Gnedin \& Ostriker (1997) the destruction time for this cluster is about 6 Hubble times, present conditions should be close to those at formation epoch.

The hypothesis that GC stars were formed in two different star formation events was intended to explain 
the presence of long blue tails in the HBs (D'Antona et al. 2002) in GCs with large abundance spread (Catelan \& de Freitas Pacheco 1995). We have shown that blue tails can be the result of the evolution of main sequence stars with larger than average (cosmological) helium abundance. These latter stars have a mass smaller than average at the giant tip at present age and, for similar mass loss along the RG branch, will have a smaller mass (and bluer location) on the horizontal branch (HB). Helium enrichment is a natural outcome of the AGB evolution (Ventura, D'Antona, \& Mazzitelli 2002), together with other chemical anomalies which can help to explain the observed abundance spreads. We leave aside the question of whether the winds from intermediate mass stars (IMS) can collect in the cluster central region, and here give origin to a new stellar generation, and concentrate on two other important issues:

1. we have to specify which initial mass function (IMF) is needed to give origin to enough stars in this second formation phase;

2. we have to explore more in detail which HB morphologies may derive from this process. In particular, the second star formation process may end when the helium content in the last ejecta (which give birth to stars) is still larger than the primordial helium content. This will produce $a$ gap in the helium content, between the original population of low mass stars, and the last born low mass stars. Does such a gap produce visible consequences in the distribution of stars in the HR diagram?

In this paper we attack these two problems, by extending the approach by D'Antona et al. (2002) to a cluster having not only a blue HB with a long blue tail, but also a red HB. We will examine the case of the cluster NGC 2808, whose HB stellar distribution shows a well populated red clump, an extended blue HB, but it contains only two RR Lyr stars. This cluster has been studied intensively in recent years. The only way to reproduce the HB distribution is apparently to invoke a bimodal (or possibly multimodal) mass distribution (e.g. Fusi Pecci \& Bellazzini 1997; Catelan et al. 1998). Differences in age or metal content among cluster members should be of a size not supported by observations. Bedin et al. (2000) make an accurate study of the reddening, differential reddening and metallicity spread in the cluster, and conclude that the $\sigma$ on $[\mathrm{Fe} / \mathrm{H}]$ is 0.03 on the Carretta \& Gratton scale (1997) and 0.05 on the Zinn \& West scale (1984). On the other hand, their CM diagram and the one by Walker (1999) leave little doubt on the matter. As for an age difference, it should be of about 2-4 Gyr as required by the jump in colour (and so in mass, about $0.04 \mathrm{M}_{\odot}$ at the cluster metallicity) across the empty RR Lyrae region: an occurrence which appears rather unlikely. Besides, as noted by Rosenberg, Recio- Blanco \& Garcia-Marin (2004), NGC 2808 is coeval with clusters of similar metallicity but much shorter HBs such as NGC 362, NGC 1261, NGC 1851. Similarly, not very much of definitive can be said on the role of rotation on HB distribution. After the many recent investigations on the matter, Sweigart (2002) concludes that: "the impact of mixing and rotation on the HB remains to be clearly established". Therefore we think important to explore new models for this complex cluster.

We here explore the possibility that the dichotomy in the HB is due to the differing helium contents of the clump stars and blue HB stars. This assumption is certainly parametric and has no pretence of uniqueness, but it has three attractive features: i) we know from the RG branch that the cluster stars show a dispersion in the abundances linked to the hot CNO cycle; ii) if we accept that the massive AGB stars are responsible for this abundance dispersion, all AGB models predict that the AGB winds are helium enriched; iii) the epoch of star formation from the AGB winds must necessarily end when massive AGBs are still evolving, and this will naturally produce a helium content gap.

It is important to stress how the large amount of information on the chemical composition of the members of GCs has changed profoundly our perception of these stellar systems. The impossibility of 
a simple solution for their formation and evolution - one star generation, a common age, one common composition - obliges to look for more complex solutions, which have to consider the detailed history of star formation, element production and dynamical evolution, and their interactions. While we can make reasonable guesses on element production, we lack any real knowledge of the dynamical history of GCs. So in this investigation we relied on a possible chemical history of NGC 2808, with only conjectures on dynamical processes.

\section{The globular cluster NGC 2808}

The HB in the CM diagram of NGC 2808 is composed by two regions clearly separated: a clump of red stars, similar to those observed in 47 Tuc, M69 and M71, and a long branch starting from the blue side of the RR Lyr gap and reaching below the cluster turnoff. In the middle, two RR Lyr variables (one of type a,b and one of type c). The (almost) absence of RR Lyr stars is in itself a surprise, but other clusters present similar HB distribution, as discussed, for example, by Catelan et al. (1998). As mentioned before, they find that the HB morphology of NGC 2808 can not be reproduced with a unimodal mass distribution, not even with a large mass dispersion, contrary to the cases of, e.g., NGC 1851 and NGC 6229. The main difficulty derives from the large amount of blue HB stars: according to Bedin et al. (2000), there are 580 blue HB members and 565 red ones ${ }^{1}$. The HR diagram from this work is shown in Fig. 1, where the red clump stars and the stars in the three different parts of the blue HB -EBT1, EBT2 and EBT3, according to Bedin et al. (2000). In terms of mass loss along the RG branch this fact would imply a strongly bimodal behaviour of the mass loss phenomenon, with no apparent reason.

The blue HB appears as a sort of "different population", which we can try to interpret in terms of selfenrichment and multiple stellar generations in GCs. On the basis of the models in Sect 3 for Z=0.001, the first star generation with $\mathrm{Y}=0.24$ and age $\sim 13$ Gyr reaches the RG tip with a mass of about 0.82 $\mathrm{M}_{\odot}$ (in absence of mass loss). The red HB clump appears centered at $(B-V)_{0} \sim 0.60$ (as shown in Fig. 1, taken from Bedin et al. (2000), assuming $\mathrm{E}(\mathrm{B}-\mathrm{V})=0.18)$, which corresponds to a HB mass of 0.68-0.71 $\mathrm{M}_{\odot}$. So the implied mass loss is of about $0.13 \mathrm{M}_{\odot}$. On the other hand, a stellar population with $\mathrm{Y}=0.28$ and age $13 \mathrm{Gyr}$ has at the $\mathrm{RG}$ tip a mass of $\sim 0.77 \mathrm{M}_{\odot}$, while for $\mathrm{Y}=0.32$ the $\mathrm{RG}$ tip mass is $\sim 0.72 \mathrm{M}_{\odot}$. With the same amount of mass loss, a mixture of these two populations would populate the region on the blue side of the RR Lyr gap, reaching $\mathrm{M}_{\mathrm{v}}$ about 1.5 mag. If we assume as mass loss law either Reimers' formulation, or any other formulation currently employed (e.g. Lee, Demarque \& Zinn 1994, Catelan 2000), the mass loss rate increases with decreasing evolving mass. The giants with $\mathrm{Y}=0.28$ and $\mathrm{Y}=0.32$ would lose about $0.034 \mathrm{M}_{\odot}$ and $0.068 \mathrm{M}_{\odot}$ more, respectively. This would allow to reach, for the case $\mathrm{Y}=0.32, \mathrm{M}_{\mathrm{v}} \sim 3.6$ : of course these are order-of-magnitude estimates, that show the potentiality of the assumptions, which will be further explored with numerical simulations.

\footnotetext{
${ }^{1}$ In this work, we use the numbers given by Bedin et al. (2000) for the different parts of the HB. These are representative of most of the HB population of the cluster. The figures plot the data kindly provided by Bedin, but include only the samples whose photometry has been obtained by HST. Therefore, e.g., the fit of the red clump stars is based on a sample of 391 stars.
} 


\section{The models}

We complemented the models of $\mathrm{Z}=0.001$ computed in paper $\mathrm{I}$ for $\mathrm{Y}=0.24$ and $\mathrm{Y}=0.28$ with models with $\mathrm{Y}=0.32$. We also evolved red giants with $\mathrm{Y}=0.32$ up to the helium flash in order to find the appropriate helium core mass for the HB models. In Figure 2 we show the evolutionary tracks of $0.82 \mathrm{M}_{\odot}$ $(\mathrm{Y}=0.24), 0.77 \mathrm{M}_{\odot}(\mathrm{Y}=0.28)$ and $0.72 \mathrm{M}_{\odot}(\mathrm{Y}=0.32)$ which evolve to the helium flash in approximately 13 Gyr. We can see that the color difference at the turnoff is negligible. A color difference of less than $0.04 \mathrm{mag}$ (corresponding to $\sim 100 \mathrm{~K}$ ) is found in the subgiant branches, while the RG branches seem to converge in color. This simple comparison shows that we do not expect to easily recognize a helium spread as large as 0.08 for clusters of this metallicity, especially if there is a dominant population with about half of the stars having $\mathrm{Y}=0.24$, while the other half are distributed in helium content, as we will show in Figure 6. Of course a helium variation can be more easily recognized in two cases:

- if in a cluster there are at least two homogeneous consistent fractions of stars with different helium abundance, the main sequences and the subgiants may show a bifurcation in colors. This might be the case shown by Bedin et al. (2004) for the main sequence of $\omega$ Cen, although the peculiarities shown by this "cluster" (multiple turnoffs and subgiant branches) depict a much more complex situation indeed;

- if the dominant population has a high helium content (this could be the case, e.g., for the clusters having a fully blue HB, namely those like M13 showing a clear "second parameter" effect), it could be recognized by its smaller $\delta(B-V)$ between the turnoff and the RG branch: e.g. a GC having normal helium would have $\delta(B-V) \simeq 0.04$ mag larger than a GC having $\mathrm{Y}=0.32$ and the same age. We mention tentatively that the different morphology of the HR diagram of M3 and M13, which has been attributed to the younger age of M3 (Rey et al. 2001), could be due to the fact that M13 stars have a higher helium abundance.

We postpone to another paper the discussion of the turnoff and main sequence luminosity and mass function in the presence of a helium spread, which requires a more detailed analysis, and may also lead to revise the mass function of low mass stars in GCs having a helium spread.

We further computed models for the evolution up to the RG branch tip and on the HB for a total heavy element content of $\mathrm{Z}=0.002$ and helium abundances $\mathrm{Y}$ of 0.24 and 0.30 . Evolutionary values for the helium core at the giant branch tip and for the helium increase in the envelope (first-dredge up) have been estimated for an age of $1310^{9} \mathrm{yr}$.

The code used is ATON2.0 (Ventura et al. 1998), and the transformation from the theoretical plane are from Bessell, Castelli, \& Plez (1998). The HB models have been evolved until the disappearance of helium in the convective core. Considering these models together with the models presented in D'Antona et al. (2002), the mass at the helium tip as function of helium and metal content can be approximated by:

$$
\begin{array}{r}
\log M_{R G} / M_{\odot} \simeq[-0.282+0.092(Y-0.24)] \times \log t \\
-1.693(Y-0.24)+2.768+12\left(Z-10^{-3}\right)
\end{array}
$$

Estimates of the heavy element content Z of NGC 2808 are between 0.001 and 0.002 (Walker 1999, Bedin et al. 2000, Carretta et al. 2003). We compared the HR diagram of the RGs and HB of NGC 2808 from Bedin et al. (2000) with our sets of models for $Z=0.001$ and $Z=0.002$. The comparison is shown in Figure 3 for $\mathrm{Z}=0.001$ : the red clump, the $\mathrm{RG}$ branch and the blue $\mathrm{HB}$ are well reproduced 
by the tracks. We adopt $\mathrm{E}(\mathrm{B}-\mathrm{V})=0.18$ (Walker 1999, Bedin et al. 2000) and $(\mathrm{m}-\mathrm{M})_{0}=15.25$. On the

contrary, the same comparison made for $\mathrm{Z}=0.002$ does not reproduce at the same time the location of the blue HB and of the RG branch, since the red giant tracks are redder by $\sim 0.08 \mathrm{mag}$ than the observed branch, once fixed the location of the blue HB. This latter depends very weakly on the metallicity and on the helium content, and is independent from the treatment of convection. The red giant branch and clump locations, on the contrary, depend on the convection model. We choose the models with $\mathrm{Z}=0.001$ on the basis of the better overall fit of the HR diagram, consistent both with the observed values of metallicity and with the estimated reddening. We examined also the case $\mathrm{Z}=0.002$; the modeling of the HB dichotomy follows the same pattern, except that the red clump requires a fine tuning of the mass loss. In any case, the results for the shape of the IMF turn out to be substantially confirmed.

For the HB, we computed masses down to $\sim 0.48 \mathrm{M}_{\odot}$, which extend to $\mathrm{T}_{\text {eff }} \sim 30000 \mathrm{~K}$. These models can not explain the hot subdwarfs which populate the most extreme clump of the HB in NGC2808. In fact, together with wCen, M54 (Rosenberg et al. 2004), and NGC6388 (Busso, Piotto \& Cassisi 2004), NGC 2808 presents the phenomenon of extremely blue, sub-luminous HB members (D'Cruz et al. 2000; Brown et al. 2001). These are objects with a luminosity lower than predicted by standard HB models, that is, models with a helium core mass determined by RG evolution terminated by the helium flash at the giant tip. Castellani \& Castellani (1993) discovered that HB structures can derive also by a helium flash taking place on the white dwarf cooling sequence, and the resulting luminosity can be up to 0.1 mag higher than found with standard models (D'Cruz et al. 1996). Still, the sub-luminous stars in $\omega$ Cen and NGC 2808 are up to 0.7 mag fainter than the standard HB, and so not understood in terms of HB structures. Sweigart (1997) suggested that a late helium flash along the white dwarf cooling sequence can penetrate inside the hydrogen burning shell, whose weakness does not provide a sufficiently strong entropy barrier (Iben 1976). The result would be a structure with an envelope rich in helium and carbon, with an effective temperature around $37000 \mathrm{~K}$ (Brown et al. 2001), while the hottest HB models lie around $31000 \mathrm{~K}$ (Castellani \& Castellani 1993; D'Cruz et al. 1996). The difference in bolometric correction and the reduced atmospheric opacity below $912 \AA$ produce the observed gap in visual magnitude. This means that these objects cannot be modeled with precision. We will assume that some of the evolving red giants suffer a delayed helium flash followed by mixing, as proposed by Sweigart (1997), and will attribute this sequence of events to all the red giants having the highest Y -and thus the smallest initial mass.

\section{The helium distribution in the HB of NGC 2808}

The mass on the HB is:

$$
M_{H B}=M_{R G}(Y, Z)-\Delta M
$$

where $M_{R G}$ is given by Eq. 1. $\Delta M$ is the average mass lost during the RG phase, with a dispersion $\sigma$ to be determined. Simple simulations show that it is possible to interpret the red part of the HB as formed by stars with helium content $\mathrm{Y}=0.24$, and the blue part as due to a population with enrichment in helium. We can derive important information by examining independently the distribution of stars in the red clump and along the blue side of the HB. 


\subsection{The red clump}

Once metallicity and age have been fixed, we adjust the average mass loss along the RG branch, and its dispersion, to fit the morphology of the HB red clump. The four panels in Fig. 4 show simulations assuming an age of 13 Gyr. Similar results are obtained for different values of the age, provided that we adopt a smaller average $\Delta M$ to reproduce the red clump, if we adopt a larger age. The dispersion assumed around $\Delta M$ is relatively small: a $\sigma \simeq 0.015 \mathrm{M}_{\odot}$ is adequate. The red clump is so compact that a larger spread in the mass loss tends to populate also the blue side of the clump, contrary to the observations. On the basis of Figure 4 we choose $\sigma=0.015 \mathrm{M}_{\odot}$.

\subsection{The blue Horizontal Branch}

Once fitted the red clump, we can suppose that the same $\Delta M$ and the same dispersion apply also to the blue side of the HB, but that the location of the stars along this branch depends on the helium content. In other words, the mass along the blue $H B$ varies mainly through the variation of $M_{R G}(Y)$, as given by Eq. 1. Thus, to obtain the lack of stars in the RR Lyr region we need to suppose that the helium content on the reddest side of the blue HB is discontinuously larger than the helium content of the red clump. One full simulation for an age of 13 Gyr is shown in Figure 5. The clump is reproduced as in Figure 4, while for the blue side we assume that the helium content is randomly distributed between $\mathrm{Y}=0.27$ and $\mathrm{Y}=0.32$. The minimum $\mathrm{Y}=0.27$ is necessary in order for the $R R$ Lyr region to remain unpopulated. In this simulation we attribute maximum observational errors of $\delta M_{\mathrm{v}}=0.025 \mathrm{mag}$ and $\delta(\mathrm{B}-\mathrm{V})=0.035 \mathrm{mag}$. Notice that our purpose here is not to obtain a "perfect" reproduction of the HR diagram, but to obtain clues on the helium variation needed to explain the blue HB.

An extra bonus of this interpretation of the HB morphology is that it gives an explanation of why the reddest stars of the blue HB are slightly more luminous than the red clump stars: in fact the different luminosities result naturally from the slight difference in the helium content of the red clump and of the red side of the blue HB. Incidentally, we remind that there are also some metal rich GCs showing a substantial blue HB population, such as NGC 6388 and NGC 6441 (Rich et al. 1997). One of the debated problems about these clusters is that the horizontal part of their HB shows a marked slope (the bluer stars being more luminous). This slope may be attributed to the same mechanism described here: the luminosity of the HB increases with $\mathrm{T}_{\text {eff }}$ just because the helium abundance of the stars increases. ${ }^{2}$ The figures also show the histogram of the number densities obtained in the simulations compared to the number densities of the blue $\mathrm{HB}$, as function of the absolute visual magnitude. Two consequences follow:

- we need some additional hypothesis to populate the faintest clump. In particular, we can suppose that some of the stars with the largest helium content (that is, with the smallest initial mass progenitor) actually follow the paths described by Sweigart (1997) and populate the hottest part of the blue HB.

- the hypothesis of a random flat distribution in helium does not reproduce well the number densities: we can then reverse the problem, as we show in the next Section.

\footnotetext{
${ }^{2}$ The anomalous distribution of the period of the RR Lyrae in NGC 6388 and NGC 6441, which does not allow to fit them into the Oosterhoff type classification (Pritzl et al. 2000), may also have the same explanation.
} 


\subsection{The progenitor masses of the matter forming the blue $\mathrm{HB}$}

We can use the relative populations along the HB to infer how many stars correspond to a given helium content, for a fixed age and average mass loss, as shown in the left side Fig. 6 for an age of $13 \mathrm{Gyr}^{3}$. We assumed an average relation between the helium content and the absolute $\mathrm{V}$ magnitude, based on the results of the simulation of Figure 5. The number of stars - helium content distribution on the left side of Figure 6 can be transformed into a relation between the number of stars now on the blue $\mathrm{HB}$ and the original AGB progenitor star mass, if we know the helium content of the ejecta as function of the progenitor mass. From the results by Ventura, D'Antona, \& Mazzitelli (2002), we adopt a simple linear relation between the average helium content in the ejecta and the progenitor mass ${ }^{4}$. We use:

$$
M_{\text {prog }}=3.5+(Y-0.26) / 0.016
$$

The right side of Fig. 6 shows the result: the blue HB contains stars whose progenitors range from $\sim 4$ to $\sim 6.5 \mathrm{M}_{\odot}$. Of course this estimate is very rough: in particular, we have made the hypothesis that the population of the extreme clump can be obtained by extrapolating the average relation between helium content and absolute visual magnitude: this produces a "helium gap" which is not real, as we have seen discussing the hottest subdwarf models in Sect. 3.

\section{Deriving a consistent initial mass function}

We should now try to understand what are the implications of Fig. 6 for the early evolution of this globular cluster. First it is necessary is to understand the mass requirements. The present day mass function of GC stars can be studied with sufficient precision only up to the turnoff, which is not larger than $0.9 \mathrm{M}_{\odot}$. We describe the IMF as made up of power laws having a form:

$$
\frac{d N}{d M}=c \times M^{-(1+x)}
$$

where $\mathrm{c}$ is a constant depending on the total mass or number of stars. In the whole range up to $0.7 \mathrm{M}_{\odot}$, Piotto \& Zoccali (1999) find that the MFs of many GCs have very mild slopes ( $\mathrm{x}$ from $\sim-0.5$ to $\sim 0.2$ ). The MF above $0.7 \mathrm{M}_{\odot}$ is steeper, and although it is difficult to determine it with care, it can be assumed close to Salpeter's slope $(\mathrm{x}=1.3)$. Kroupa (2001) makes the case for an universal IMF for stellar systems of different metallicities, at intermediate and high masses. His reassessment of the IMF is made for

\footnotetext{
${ }^{3}$ While the total number of stars in the red clump is comparable to the number of stars in the blue HB, it is evident that the sample having the cosmological helium is prominent, in comparison with the distribution of the objects with larger helium. For this reason, other evidences for differing helium content among the stars in this cluster are not easy to be traced: in particular 1) the location of the red giant bump (e.g. Zoccali et al. 1999), which also depends on the helium abundance, will be dominated by the red giants having a normal helium; 2) the parameter R, namely the ratio between the number of HB stars and the number of RGs above the HB level, is not much affected, as the level of the HB is taken at the red side, populated by stars with normal helium, and the evolutionary times of the blue HB stars do not change in a sensible way with $\mathrm{Y}$.

${ }^{4}$ Actually the helium yields of AGB stars plotted in Fig. 4 of Ventura, D'Antona, \& Mazzitelli (2002) show a monotonic, but non linear behaviour as function of the progenitor mass, and even this characteristic could have a role in producing a clumpy distribution of the blue HB in this or in other GCs. Nevertheless, it is more instructive to simplify the issue, until we have a better understanding of the problem.
} 
many stellar populations and different mass ranges - and shows that the typical IMF index is $x \simeq 1.3$, that is Salpeter's index, or a larger value, while the small masses have much milder slopes. Simplifying Kroupa's results, we describe the IMF of GCs as made up of a power law with index x=0 below $0.9 \mathrm{M}_{\odot}$, connected to a power law with index $\mathrm{x}=1.3$ or $\mathrm{x}=2$ above a "transition mass" $\mathrm{M}_{\text {trans }}=0.9 \mathrm{M}_{\odot}$.

If NGC 2808 is made up by a first stellar population having standard helium content $\mathrm{Y}=0.24$, from which, today, the stars populating the red clump of the HB evolve (and not considering for the moment the loss of stars from the cluster), the number of clump stars provide a point of calibration of the IMF. We can write:

$$
N_{\text {redHB }}=\left(\frac{d N}{d M}\right)_{M_{R G}} \times\left(\frac{d M}{d t}\right)_{M_{R G}} \times \tau_{H B}
$$

where $\tau_{H B}$ is the typical evolutionary time in the HB phase. From Eq. 1 we can derive the time derivative of the mass $M_{R G}$

$$
\left(\frac{d M}{d t}\right)_{M_{R G}}=-0.282 \times M_{R G} / t_{G C}
$$

where $t_{G C}$ is the age of the cluster. As expected, the fraction of stars on the HB is proportional to the ratio $\tau_{H B} / t_{G C}$. At the mass today evolving on the RGB, given by Eq. 1, and fixing an age of $13 \mathrm{Gyr}$, we can calibrate the coefficient in the IMF of Eq. 4. The resulting global IMFs, satisfying this constraints and the change of slope at $\mathrm{M}_{\text {trans }}=0.9 \mathrm{M}_{\odot}$, are shown by the dash-dotted lines in Fig. 7 .

Let us assume that indeed all the mass lost by the intermediate mass stars can form other stars. Their IMF must have a slope which is at least Salpeter's slope, in the hypothesis that Kroupa's results can be representative also of the IMF of stars of mass $M>M_{\text {trans }}$ for the primitive GC. The main feature to be explained is EBT1, the most populated clump in the blue HB, which, for an age of 13 Gyr, and average mass loss during the $\mathrm{RG}$ phase of $0.13 \mathrm{M}_{\odot}$, corresponds to progenitor masses from $\mathrm{M}_{\text {prog } 1}=4.1$ to $\mathrm{M}_{\text {prog } 2}=5 \mathrm{M}_{\odot}$. In the sample by Bedin et al. (2000) these stars are $N_{B H B}=401$. To understand how much mass we need from these progenitors, we have now to make a hypothesis about the distribution of stellar masses in this new stellar population coming out from the ejecta. It is difficult to infer anything about the formation -if any- of massive stars, but we can make the reasonable hypothesis that, at low masses, this MF must again resemble the MF which we see today in GCs: $\mathrm{x}=0$ for $M<M_{\text {trans } 2}, \mathrm{x}=1.3$ for $M>M_{\text {trans } 2}$, and $M_{\text {trans } 2}=0.9 \mathrm{M}_{\odot}$. We further make the hypothesis that star formation in this case is limited to stars not more massive than $1.5 \mathrm{M}_{\odot}$. From this sample, some stars are evolving today in the main blue clump EBT1. The expected number is given by Eq. 5, where we now consider the number of stars in EBT1, $N_{B H B}=401$. The derivative $d M / d t$ is now slightly different:

$$
\frac{d M}{d t}=[-0.282+0.092(Y-0.24)] \times M_{R G} / t_{G C}
$$

We use $Y=0.28$, which is the average value for this population (see Fig. 6). In this way, we calibrate the coefficients which enter in the IMF of the second stellar generation, and establish which is the total mass which must be locked into the second generation stars in order to form the appropriate number of blue HB stars.

When we calibrate the IMF of the upper mass range of the first stellar population, we find that the mass function at masses 4.1-5 must be much larger than predicted if the IMF of the GC were "normal", as described above.

In order to fit both the red clump IMF and the intermediate mass stars IMF, we need to use two very different slopes: we adopt Salpeter's slope (or a larger slope) for the masses which include our normalization point at $4.1-5 \mathrm{M}_{\odot}$, and slopes which can fit the red clump for small masses. This defines 
a $\mathrm{M}_{\text {trans }}$, where the two IMFs must coincide. Some results are shown in Fig. 7, and in Table 1. Finally, it is possible to satisfy both constraints by using a unique IMF. In this case it must have a slope $\mathrm{x}=-0.14$, as we see in Table 1 . Such an IMF can not be extrapolated too much, as it predicts an unrealistic high mass for the cluster and is not consistent with all the evidence concerning the IMFs derived from many different stellar environments.

\subsection{The extreme blue tails 2 and 3}

Although the number of stars in the EBT1 provides the best constraint to our IMF, we can try to use the number of stars in the EBT2 and EBT3 together, to infer another point of the star formation rate of the first stellar generation. Of course the relation between progenitor mass and helium content becomes more uncertain here, as we do not know well the evolutionary meaning of the EBT3 stars. We can consider two cases: either these stars come from progenitor masses between 5.1 and $6.6 \mathrm{M}_{\odot}$ (as indicated in Fig 6), or we assign to all of them the mass range $5.1-6 \mathrm{M}_{\odot}$ that is, we attribute all stars to the mass range of the EBT2, and consider the EBT3 clump as due to random occurrence of the flash-induced mixing suggested by Sweigart (1997). The corresponding mass function points are given in Fig 7. We see that in both cases the mass function is well below the predictions based on the point relative to EBT1, and assuming IMF slopes up to $\mathrm{x}=2$. We do not give too much weight to this result, but at face value it indicates that the second generation star formation is less favoured at higher AGB masses. This may be due, e.g., to the last supernova events which take place into the cluster, when already the most massive AGB stars are evolving.

\section{Discussion}

Figure 7 summarizes the schematic IMFs based on our interpretation of the morphology of the HB in NGC 2808. The result has been obtained by many simplifying assumptions, but a variation of the input hypotheses does not produce a qualitatively different behaviour, as we have also checked by assuming a different global metallicity for the cluster.

1) Let us assume that the original IMF of NGC 2808 is similar to the "universal" IMF by Kroupa (2001), in a simplified form: two power laws with indices $\mathrm{x}=0$ below $0.9 \mathrm{M}_{\odot}$ and $\mathrm{x}=1.3$ above it. In this case all the HB stars must come from only one population, as the matter lost by IMS is short by a factor 10 or so from being able to form the population which we see today in the blue HB. A similar conclusion was reached a long time ago by Cottrell \& Da Costa (1981) and was in fact the most severe problem in any kind of self-enrichment scheme for GCs - apart from the self-pollution by accretion on stars proposed by D'Antona, Gratton, \& Chieffi (1983).

2) In order to have enough stars in the main blue clump EBT1, we need a relatively flat IMF, that is $\mathrm{x} \sim 0$. But is there any evidence for a flat IMF up to the mass range of massive AGBs, that is stars of $4-7 \mathrm{M}_{\odot}$ ? A possible solution is that the IMF slope in the central parts of clusters is indeed different from that in the field. Panagia et al. (2000), in a systematic study of the young stellar population around the Supernova 1987A, show that the number ratio of low mass $\left(1-2 \mathrm{M}_{\odot}\right)$ stars to massive stars is very low, $\sim 1.4$, within 130 '. from the supernova, corresponding to about 30pc, while it is $\sim 8.9$ in the general field: this translates in an IMF slope $\mathrm{x}=0.5$, to be compared with $\mathrm{x}=1.5$ for the entire field. An IMF of mild slope is therefore not to be ruled out: it may well be that in compact stellar systems the IMF slope 
Table 1. IMF and Mass of the primordial Globular Cluster NGC 2808

\begin{tabular}{crrrcccc}
\hline \hline $\mathrm{M}_{\text {tot }}^{(a)}$ & $\mathrm{M}_{\text {trans }}$ & \multicolumn{1}{c}{$\mathrm{x}_{\text {low }}$} & \multicolumn{1}{c}{$\mathrm{x}_{\text {up }}$} & $\left.\mathrm{N}_{\text {tot }}^{(a}\right)$ & $\mathrm{N}_{N S}$ & $\mathrm{~N}_{B H}$ & $\mathrm{M}_{\text {initial }}^{(b)}$ \\
\hline $1.01 \mathrm{E}+06$ & 0.90 & 0.00 & 1.30 & $1.04 \mathrm{E}+06$ & $1.40 \mathrm{E}+04$ & $3.90 \mathrm{E}+03$ & $1.01 \mathrm{E}+06$ \\
$5.58 \mathrm{E}+05$ & 0.90 & 0.00 & 2.00 & $9.52 \mathrm{E}+05$ & $2.62 \mathrm{E}+03$ & $3.16 \mathrm{E}+02$ & $5.58 \mathrm{E}+05$ \\
$5.37 \mathrm{E}+07$ & 5.00 & -0.14 & -0.14 & $2.78 \mathrm{E}+06$ & $4.99 \mathrm{E}+05$ & $8.82 \mathrm{E}+05$ & $5.37 \mathrm{E}+07$ \\
\hline $7.77 \mathrm{E}+06$ & 3.03 & -0.50 & 1.01 & $1.65 \mathrm{E}+06$ & $1.86 \mathrm{E}+05$ & $7.39 \mathrm{E}+04$ & $1.04 \mathrm{E}+07$ \\
$8.07 \mathrm{E}+06$ & 2.20 & -0.99 & 1.01 & $1.68 \mathrm{E}+06$ & $1.86 \mathrm{E}+05$ & $7.39 \mathrm{E}+04$ & $1.04 \mathrm{E}+07$ \\
$8.26 \mathrm{E}+06$ & 1.80 & -1.50 & 1.01 & $1.73 \mathrm{E}+06$ & $1.86 \mathrm{E}+05$ & $7.39 \mathrm{E}+04$ & $1.04 \mathrm{E}+07$ \\
$8.38 \mathrm{E}+06$ & 1.58 & -2.00 & 1.01 & $1.78 \mathrm{E}+06$ & $1.86 \mathrm{E}+05$ & $7.39 \mathrm{E}+04$ & $1.04 \mathrm{E}+07$ \\
$8.47 \mathrm{E}+06$ & 1.44 & -2.50 & 1.01 & $1.82 \mathrm{E}+06$ & $1.86 \mathrm{E}+05$ & $7.39 \mathrm{E}+04$ & $1.04 \mathrm{E}+07$ \\
\hline $5.86 \mathrm{E}+06$ & 3.23 & -0.50 & 1.30 & $1.58 \mathrm{E}+06$ & $1.47 \mathrm{E}+05$ & $4.08 \mathrm{E}+04$ & $1.01 \mathrm{E}+07$ \\
$6.26 \mathrm{E}+06$ & 2.41 & -0.99 & 1.30 & $1.64 \mathrm{E}+06$ & $1.47 \mathrm{E}+05$ & $4.08 \mathrm{E}+04$ & $1.01 \mathrm{E}+07$ \\
$6.54 \mathrm{E}+06$ & 1.98 & -1.50 & 1.30 & $1.74 \mathrm{E}+06$ & $1.47 \mathrm{E}+05$ & $4.08 \mathrm{E}+04$ & $1.01 \mathrm{E}+07$ \\
$6.74 \mathrm{E}+06$ & 1.74 & -2.00 & 1.30 & $1.83 \mathrm{E}+06$ & $1.47 \mathrm{E}+05$ & $4.08 \mathrm{E}+04$ & $1.01 \mathrm{E}+07$ \\
$6.89 \mathrm{E}+06$ & 1.57 & -2.50 & 1.30 & $1.92 \mathrm{E}+06$ & $1.47 \mathrm{E}+05$ & $4.08 \mathrm{E}+04$ & $1.01 \mathrm{E}+07$ \\
\hline $3.91 \mathrm{E}+06$ & 3.55 & -0.50 & 2.00 & $1.49 \mathrm{E}+06$ & $8.48 \mathrm{E}+04$ & $1.02 \mathrm{E}+04$ & $1.63 \mathrm{E}+07$ \\
$4.50 \mathrm{E}+06$ & 2.79 & -0.99 & 2.00 & $1.62 \mathrm{E}+06$ & $8.48 \mathrm{E}+04$ & $1.02 \mathrm{E}+04$ & $1.63 \mathrm{E}+07$ \\
$5.02 \mathrm{E}+06$ & 2.34 & -1.50 & 2.00 & $1.81 \mathrm{E}+06$ & $8.48 \mathrm{E}+04$ & $1.02 \mathrm{E}+04$ & $1.63 \mathrm{E}+07$ \\
$5.46 \mathrm{E}+06$ & 2.05 & -2.00 & 2.00 & $2.03 \mathrm{E}+06$ & $8.48 \mathrm{E}+04$ & $1.02 \mathrm{E}+04$ & $1.63 \mathrm{E}+07$ \\
$5.83 \mathrm{E}+06$ & 1.85 & -2.50 & 2.00 & $2.24 \mathrm{E}+06$ & $8.48 \mathrm{E}+04$ & $1.02 \mathrm{E}+04$ & $1.63 \mathrm{E}+07$ \\
\hline $3.10 \mathrm{E}+06$ & 3.80 & -0.50 & 3.00 & $1.43 \mathrm{E}+06$ & $4.13 \mathrm{E}+04$ & $1.59 \mathrm{E}+03$ & $5.46 \mathrm{E}+07$ \\
$3.88 \mathrm{E}+06$ & 3.15 & -0.99 & 3.00 & $1.62 \mathrm{E}+06$ & $4.13 \mathrm{E}+04$ & $1.59 \mathrm{E}+03$ & $5.46 \mathrm{E}+07$ \\
$4.73 \mathrm{E}+06$ & 2.71 & -1.50 & 3.00 & $1.94 \mathrm{E}+06$ & $4.13 \mathrm{E}+04$ & $1.59 \mathrm{E}+03$ & $5.46 \mathrm{E}+07$ \\
$5.56 \mathrm{E}+06$ & 2.40 & -2.00 & 3.00 & $2.31 \mathrm{E}+06$ & $4.13 \mathrm{E}+04$ & $1.59 \mathrm{E}+03$ & $5.46 \mathrm{E}+07$ \\
$6.37 \mathrm{E}+06$ & 2.18 & -2.50 & 3.00 & $2.73 \mathrm{E}+06$ & $4.13 \mathrm{E}+04$ & $1.59 \mathrm{E}+03$ & $5.46 \mathrm{E}+07$ \\
\hline
\end{tabular}

${ }^{a}$ Mass and total number of stars included between $0.08 \mathrm{M}_{\odot}$ and $100 \mathrm{M}_{\odot}$, using the IMF indices of column 3 and 4

${ }^{\mathrm{b}}$ Mass which the cluster had, if it were constrained by the total wind mass between 4.1 and $5 \mathrm{M}_{\odot}$, but the $\mathrm{IMF}$ extends till $\mathrm{M}=0.9 \mathrm{M}_{\odot}$ with index $x_{\text {up }}$, changing to $x_{\text {low }}=0$ below $\mathrm{M}=0.9 \mathrm{M}_{\odot}$. 
is mild, while it is much steeper in the general field, reflecting different processes of star formation, as Panagia et al. (2000) suggest. Other hints came some years ago based on the possible interpretation of MACHO halo objects in terms of halo white dwarfs (Adams \& Laughlin 1996; Chabrier et al. 1996). The same Kroupa (2001) suggests a systematically varying IMF, which, in the past, had a characteristic fragmentation mass around $1-2 \mathrm{M}_{\odot}$, while today it is about $0.1 \mathrm{M}_{\odot}$.

3) Actually, the process of formation of a second generation of stars can not be decoupled from the dynamical evolution of the cluster: during the first phases of life, the IMS had time to segregate into the cluster central parts, where, for this reason, indeed the IMF would look like those shown in Fig. $7^{5}$. The ejecta of these stars gave birth to the second generation, but the external parts of the cluster, containing all the other first generation low mass stars, was lost, leaving the present proportion of first and second generation stars. The IMFs shown in Fig. 7 can be considered then simple examples of what actually should be obtained by considering the proper dynamic evolution of the cluster, including the loss of low mass stars from it. What seems to be clear is that, if the true IMF of the first stellar population of GCs was similar to the field IMF, we need that most of the low mass stars, today progenitors of the HB red clump, has been lost from the cluster.

\subsection{The neutron stars and black holes from the first generation}

Table 1 lists some properties derived from the IMFs shown in Fig. 7, which refer to an age of 13Gyr, an average mass loss of $0.13 \mathrm{M}_{\odot}$ and a $\sigma$ of $0.015 \mathrm{M}_{\odot}$. In particular, we see that the initial mass of NGC 2808 must have been much larger indeed than the mass we see today. Of course, this work does not enter into the problems of loss of stability of the cluster, which however will be a powerful constraint on the reliability of each proposed IMF. We wish to point out an additional possible consequence of our interpretation of the HB of NGC 2808: it provides an appealing hint for the solution of a problem in GC stellar content: the amount of neutron stars (NS) and black holes (BH).

We can formally compute the number of NSs and BHs for each of the IMFs which satisfy the constraint posed by the blue HB, in the hypothesis that we are authorized to extrapolate their number to the mass range of NS and $\mathrm{BH}$ progenitors. The total number of NS is obtained by considering the progenitors between the upper mass which gives origin to AGB stars $\left(7 \mathrm{M}_{\odot}\right)$ and $25 \mathrm{M}_{\odot}$, and $\mathrm{BH}$ formation is considered between 25 and $100 \mathrm{M}_{\odot}$.

The large number of millisecond pulsars (MSPs) discovered in recent years in many GCs, and in particular the 20 MSPs discovered in the cluster 47 Tuc (Camilo et al. 2000) indicate that the richest GCs may contain more than $\sim 1000$ NSs (e.g., Pfahl et al. 2003) a number embarassingly large. In fact, the number of NSs predicted by a normal IMF in NGC 2808 would be between 2600 and 14000 (top of Table 1), implying a retention factor from 7 to 40\%, at variance with the characteristic kick speeds of single radiopulsar in the Galaxy, which have an average $<v>\sim 250-300 \mathrm{Km} / \mathrm{s}$ (Hansen \& Phinney 1997), much larger than the escape velocity from typical clusters ( 25Km/s). Pfahl et al. (2003) have shown that the retention factor is between $1 \%$ and $8 \%$, even including consideration of binariety, but it would have been much smaller if they had taken into account a number of other effects. Table 1 shows that the retention problem becomes less severe if we assume that the IMF of intermediate mass stars,

\footnotetext{
${ }^{5}$ Indeed, for a young GC belonging to the Large Magellanic Cloud, NGC 1866, which has an age close to the age for which we expect the second stage of star formation to have been efficient in GCs, Brocato et al. (2003) find a strong evidence for mass segragation in the cluster core, for masses $\sim 4 \mathrm{M}_{\odot}$.
} 
necessary to interpret the blue HB morphology, can be extended to the masses which form neutron stars, even if the slope is much larger than Salpeter's (up to $\mathrm{x}=2$ ).

The presence of stellar or intermediate mass BHs in Globular Clusters is exciting today a renewed interest. On the one side, recent observations by STIS on HST of the velocity dispersion in the center of the cluster M15 seem to indicate the possible presence of a massive BH (a few thousand $\mathrm{M}_{\odot}$ ) in the core (Gerssen et al. 2002). On the other hand, the presence of an intermediate mass BH, or of binary BH systems, may explain the peculiar dynamical behaviour of the MSPs in the cluster NGC 6752 (D'Amico et al. 2002), and in particular the location of PSR A at more than 3 half-mass radii from the center (Colpi, Mapelli, \& Possenti 2003).

According to Table 1, an important number of stellar mass black holes could have been formed in the cluster, with interesting consequences on its further dynamical evolution. BHs should have minor retention problems, as the galactic distribution of black hole X-ray binaries has a mean velocity of only $\sim 40$ $\mathrm{km} \mathrm{s}^{-1}$ (White \& van Paradijs 1996), implying no or negligible kicks. However, close (three- or fourbody) dynamical encounters among BHs, may expell them from clusters, and in fact current N-body simulations (Portegies Zwart \& McMillan 2000) foresee no black hole or at most one black hole binary to remain in the cluster. In addition, even less is known about their detailed formation (see, e.g. Fryer \& Kalogera 2001), and we have inferred their possible numbers by extrapolating the IMF to masses an order of magnitude larger than our calibration point at $\sim 5 \mathrm{M}_{\odot}$. The numbers in Table 1 , therefore, should be taken as helpful indication, and certainly not as predictions.

As a final caveat, notice that the interaction between the AGB winds and the stellar mass BHs possibly present in the central regions is not devoid of consequences, either as a help to the star formation mechanism, or as a sink of gas: the problem of the early evolution of GCs is all to be worked out.

\section{Conclusions}

In this paper we have used the galactic GC NGC 2808 as a laboratory to experiment on recent ideas on the early evolution of GCs. The HB in this cluster shows a very peculiar morphology: it is well populated in the red clump and in the blue and extremely blue region, but almost devoid of stars in the RR Lyr region. This morphology can be understood in terms of the coexistence of two stellar generations, separated by a difference between the helium content of the first one and the minimum helium content of the second one $(\Delta Y \simeq 0.03)$. The small luminosity difference between the red and blue side of the horizontal part of the $\mathrm{HB}$ is also explained by this helium difference.

The peculiar HB morphology of NGC 2808 is therefore consistent with the hypothesis that the stars in many GCs were formed in two different events, a hypothesis primarily invoked to explain the abundance spreads in the light elements involved in the hot CNO cycle (Cottrell \& Da Costa 1981; D'Antona et al. 2002). Using an average relation between the progenitor AGB mass and the helium content of its ejecta and the HB data by Bedin et al. (2000), we derive two points of the IMF of the cluster stars: 1) the point at $\mathrm{M}=0.82 \mathrm{M}_{\odot}$, that is the mass function value for the stars which today are found in the red clump, having the primordial helium content; 2) the point for the mass range $\sim 4.1-5 \mathrm{M}_{\odot}$, whose ejecta should populate the most populous clump of the blue HB. Although this second point is somewhat model dependent, it clearly results that we need many more AGB stars than predicted by an IMF similar to those inferred for many stellar environments (Kroupa 2001). We favour then the possibility that many of the low mass stars of the first stellar generation have been lost, but a dynamical study of these first stages of star formation is needed.

We comment on the large number of NSs - and possibly also BHs- which our model predict, and which 
would make easier to understand the population of millisecond pulsars in many GCs and the possible presence of intermediate mass BHs in the clusters M15 and NGC 6752.

As for possible checks of the proposed scenario, the disappearance of surface helium (through sedimentation) at temperatures at which it would become observable, makes direct measurements impossible. A way to check the multiple star generations hypothesis could be the accurate measurement of main sequence masses in double stars, as well as the detailed analysis of the behaviour of various CM diagram features, such as the magnitude difference between the RG branch bump and the turnoff.

We thank all the GC italian friends who helped us in collecting data and ideas, and mainly R. Gratton, G. Piotto, M. Zoccali and L.R. Bedin. 'Rolly' Bedin is also thanked for a critical reading of the manuscript. F.D. thanks A. Possenti and M. Colpi for useful discussion about the neutron star and black hole content of galactic Globular Clusters. Comments by an anonymous referee helped us to clarify the proposed scenario.

\section{REFERENCES}

Adams, F.. C. \& Laughlin, G. 1996, ApJ, 468, 586

Bedin, L. R., Piotto, G., Zoccali, M., Stetson, P. B., Saviane, I., Cassisi, S., \& Bono, G. 2000, A\&A, 363,159

Bedin, L. R., Piotto, G., Anderson, J., Cassisi, S., King, I. R., Momany, Y., Carraro, G. 2004, ApJ, 605, L125

Bessell, M. S., Castelli, F., \& Plez, B. 1998, A\&A, 333, 231

Brocato, E., Castellani, V., Di Carlo, E., Raimondo, G., \& Walker, A. R. 2003, AJ, 125, 3111

Brown, T. M., Sweigart, A. V., Lanz, T., Landsman, W. B., \& Hubeny, I. 2001, ApJ, 562, 368

Busso, G., Piotto, G., \& Cassisi, S. 2004, Memorie della Societa Astronomica Italiana, 75, 46

Camilo, F., Lorimer, D. R., Freire, P., Lyne, A. G., \& Manchester, R. N. 2000, ApJ, 535, 975

Carretta E., \& Gratton R.G. 1997, A\&AS, 121, 95

Carretta, E., Bragaglia, A., Cacciari, C., \& Rossetti, E. 2003, A\&A, 410, 143

Castellani. M, \& Castellani V. 1993, ApJ, 407, 649

Catelan, M., \& de Freitas Pacheco, J. A. 1995, A\&A, 297, 345

Catelan, M., Borissova, J., Sweigart, A. V., \& Spassova, N. 1998, ApJ, 494, 265

Catelan, M. 2000, ApJ, 531, 826

Chabrier, G., Segretain, L., \& M'era, D. 1996, ApJ, 468, L21

Colpi, M., Mapelli, M., \& Possenti, A. 2003, ApJ, 599, 1260 
Cottrell, P. L. \& Da Costa, G. S. 1981, ApJ, 245, L79

D’Amico, N., Possenti, A., Fici, L., Manchester, R. N., Lyne, A. G., Camilo, F., \& Sarkissian, J. 2002, ApJ, 570, L89

D’Antona, F., Gratton, R., \& Chieffi, A. 1983, Memorie della Societa Astronomica Italiana, 54, 173

D’Antona, F., Caloi, V., Montalbán, J., Ventura, P., \& Gratton, R. 2002, A\&A, 395, 69

D’Antona, F. 2003, Societa Astronomica Italiana Memorie Supplement, 3, 64

D’Cruz N.L., Dorman B., Rood R.T., \& O’Connell R.W. 1996, ApJ, 466, 359

D’Cruz, N. L. et al. 2000, ApJ, 530, 352

Denissenkov, P. A. \& Herwig, F. 2003, ApJ, 590, L99

Freeman, K. C.; Rodgers, A. W. 1975, ApJ, 201, L71

Freeman, K. C.; Norris, J. 1981, ARA\&A, 19, 319

Fryer, C. L. \& Kalogera, V. 2001, ApJ, 554, 548

Fusi Pecci, F. \& Bellazzini, M. 1997, The Third Conference on Faint Blue Stars, 255

Gerssen, J., van der Marel, R. P., Gebhardt, K., Guhathakurta, P., Peterson, R. C., \& Pryor, C. 2002, AJ, 124, 3270 (addendum 125, 376 [2003])

Gnedin, O. Y. \& Ostriker, J. P. 1997, ApJ, 474, 223

Gnedin, O. Y., Zhao, H., Pringle, J. E., Fall, S. M., Livio, M., \& Meylan, G. 2002, ApJ, 568, L23

Gratton, R. G. et al. 2001, A\&A, 369, 87

Gratton, G., Sneden, C., \& Carretta, E. 2004, ARA\&A, in press

Hansen, B. M. S. \& Phinney, E. S. 1997, MNRAS, 291, 569

Harris, W. E. 1996, VizieR Online Data Catalog, 7195

Iben, I.Jr. 1976, ApJ, 208,165

Kraft, R. P. 1994, PASP, 106, 553

Kroupa, P. 2001, MNRAS, 322, 231

Lee, Y., Demarque, P., \& Zinn, R. 1994, ApJ, 423, 248

Loup, C., Forveille, T., Omont, A., \& Paul, J. F. 1993, A\&AS, 99, 291

Mallia, E. A. \& Pagel, B. E. J. 1981, MNRAS, 194, 421

Panagia, N., Romaniello, Scuderi \& Kirshner 2000, ApJ 539, 197 
Pfahl, E., Rappaport, S. \& Podsiadlowski, P. 2003, ApJ, 573, 283

Piotto, G. \& Zoccali, M. 1999, A\&A, 345, 485

Portegies Zwart, S. F. \& McMillan, S. L. W. 2000, ApJ, 528, L17

Pryor, C. \& Meylan, G. 1993, in Structure and Dynamics of Globular Clusters, S.G. Djorgovsky and G. Meylan eds., ASP Conference Series, 50, 357

Pritzl, B., Smith, H. A., Catelan, M., \& Sweigart, A. V. 2000, ApJ, 530, L41

Rey, S., Yoon, S., Lee, Y., Chaboyer, B., \& Sarajedini, A. 2001, AJ, 122, 3219

Rich, R. M. et al. 1997, ApJ, 484, L25

Rosenberg, A., Recio-Blanco, A., \& García-Marín, M. 2004, ApJ, 603, 135

Smith G. H. 1987, PASP, 99, 67

Smith, V. V., Suntzeff, N. B., Cunha, K., Gallino, R., Busso, M., Lambert, D. L., \& Straniero, O. 2000, AJ, 119, 1239

Sneden C. 1999, Ap\&SS, 265, 145

Sneden C. 2000, in 35th Liege International Astrophysics Colloquium: The Galactic Halo, from Globular Clusters to Field Stars, ed. A. Noels, P. Magain, D. Caro, E. Jehin, G. Parmentier, and A. Thoul (Liège Belgium: Institut d'Astrophysique et de Géophysique), p. 159

Sweigart, A. V. 1997, Third Conference on Faint Blue Stars, ed. A. G. D. Philip, J. Liebert, R. Saffer and D.S. Hayes, (Schenectady, L. Davis Press), p.3

Sweigart, A. V. 2002, Highlights of Astronomy, Vol. 12, p. 292

Thoul, A., Jorissen, A., Goriely, S., Jehin, E., Magain, P., Noels, A., \& Parmentier, G. 2002, A\&A, 383, 491

Ventura, P., Zeppieri, A., Mazzitelli, I., \& D’Antona, F. 1998, A\&A, 334, 953

Ventura, P., D’Antona, F., Mazzitelli, I., \& Gratton, R. 2001, ApJ, 550, L65

Ventura, P., D’Antona, F., \& Mazzitelli, I. 2002, A\&A, 393, 215

Ventura, P., Mazzitelli, I. \& D'Antona, F. 2004, Proceedings of the Joint Discussion 04 of the XXIV IAU General Assembly, Sydney, Astrophysical Impact of Abundances in Globular Cluster Stars, eds. F.D'Antona and G. Da Costa, Mem.S.A.It. 75, n. 2, in presse

Walker, A. R. 1999, AJ, 118, 432

White, N. E. \& van Paradijs, J. 1996, ApJ, 473, L25

Zinn R., \& West M.J. 1984, ApJS, 55, 45

Zoccali, M., Cassisi, S., Piotto, G., Bono, G., \& Salaris, M. 1999, ApJ, 518, L49 


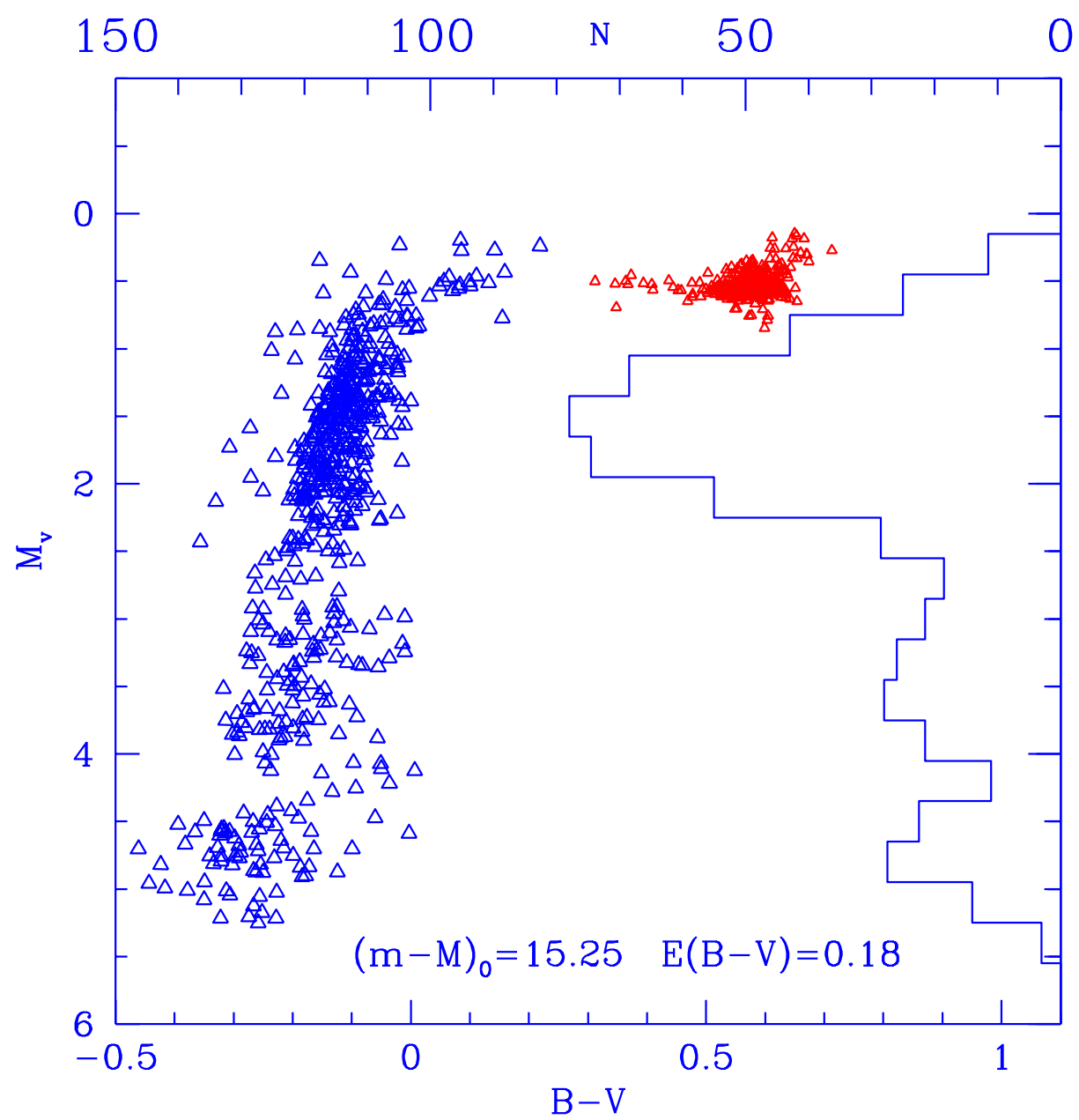

Fig. 1. - The HB in the HST data by Bedin et al. (2000) for the GC NGC 2808. It consists of a red clump plus a composite blue part, along which two main gaps are well evident. The histogram (scale given on the top abscissa) represents the number of stars in the blue part of the $\mathrm{HB}$, as function of $\mathrm{M}_{\mathrm{v}}$. 


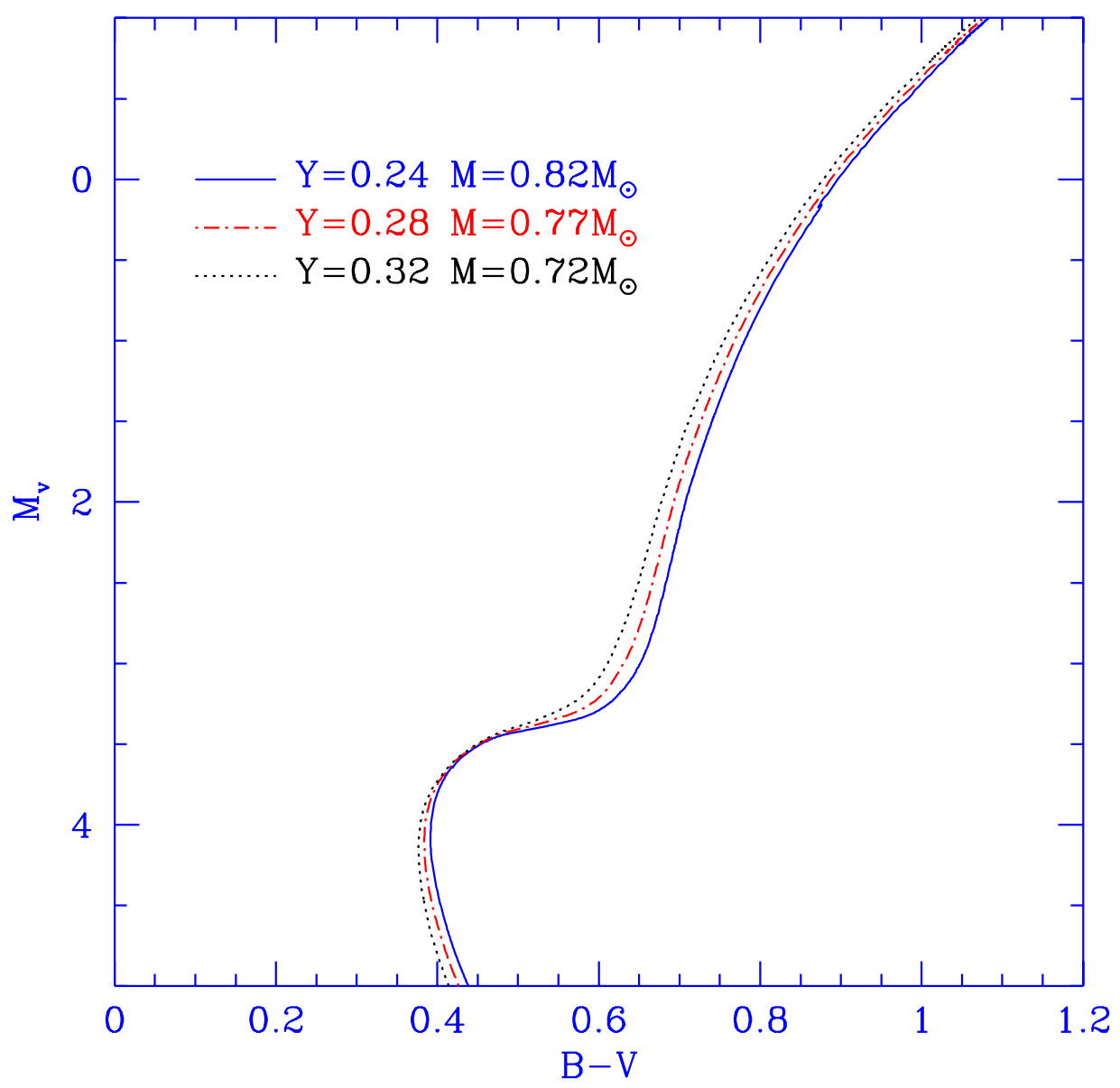

Fig. 2.- Tracks of different helium content, corresponding to an average age of $13 \mathrm{Gyr}$ at the giant branch, are compared in the observational plane. The maximum difference in the location occurs at the subgiant branch $(\sim 0.04 \mathrm{mag})$. The turnoff location is not sensibly affected, while the main sequence shows again a color difference of few hundreths of magnitude. 


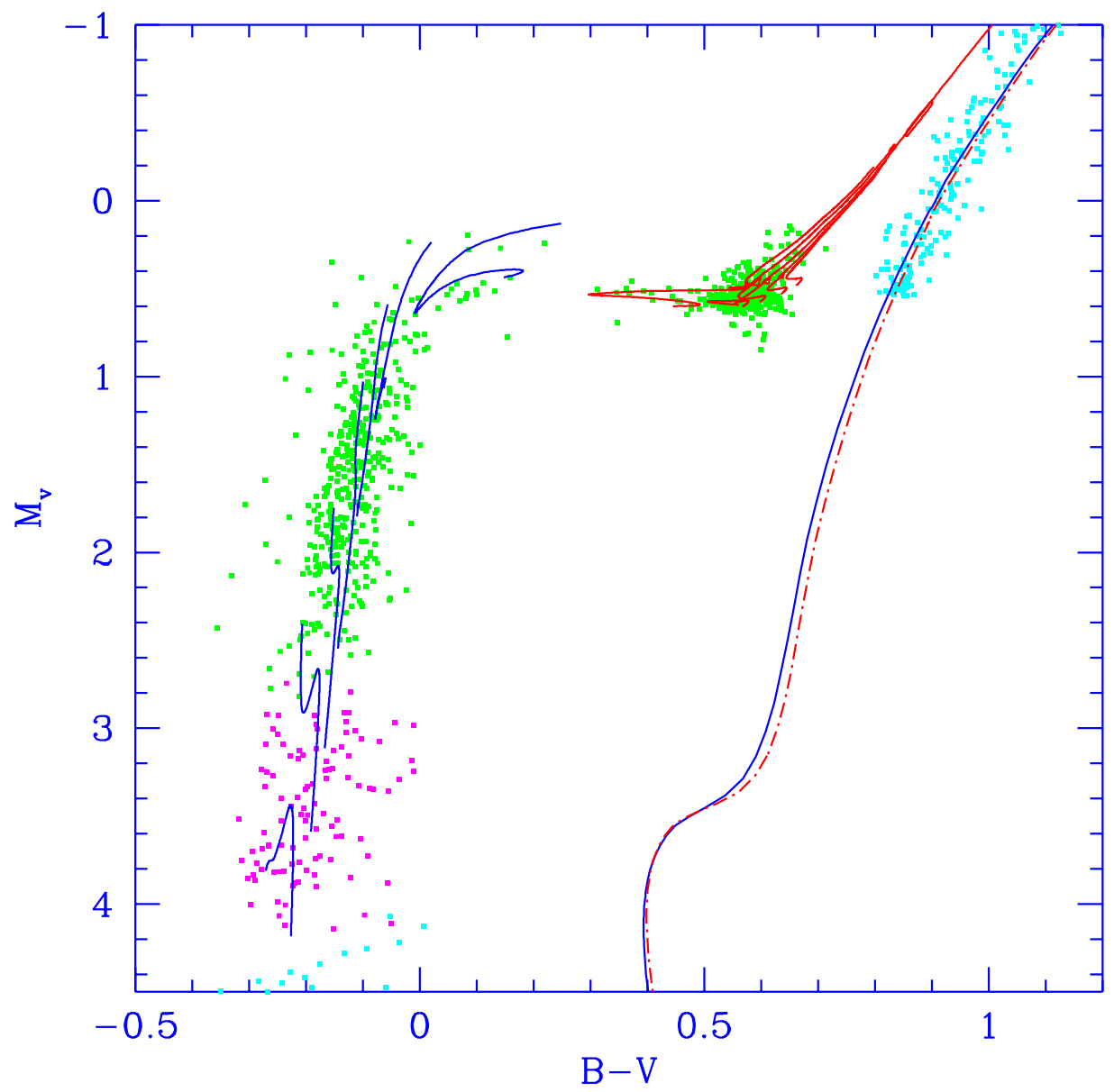

Fig. 3. - The models are compared with the observations of the cluster stars (distance modulus (m$\mathrm{M})_{0}=15.25$ and $\left.\mathrm{E}(\mathrm{B}-\mathrm{V})=0.18\right)$. The turnoff and red giant branch isochrones are shown for $13 \mathrm{Gyr}$ and for $\mathrm{Y}=0.24$ and $\mathrm{Y}=0.28$. The tracks on the red clump are for masses $\mathrm{M}=0.66,0.68,0.70,0.74$ and $0.8 \mathrm{M}_{\odot}$, $\mathrm{Y}=0.24$. The tracks on the blue are for $\mathrm{M}=0.50,0.52,0.54,0.56,0.58,0.60$ and $0.63 \mathrm{M}_{\odot}$, starting from the lowest one. The extreme blue clump (not shown in the figure) is touched by models of $0.48 \mathrm{M}_{\odot}($ but see discussion in the text). 


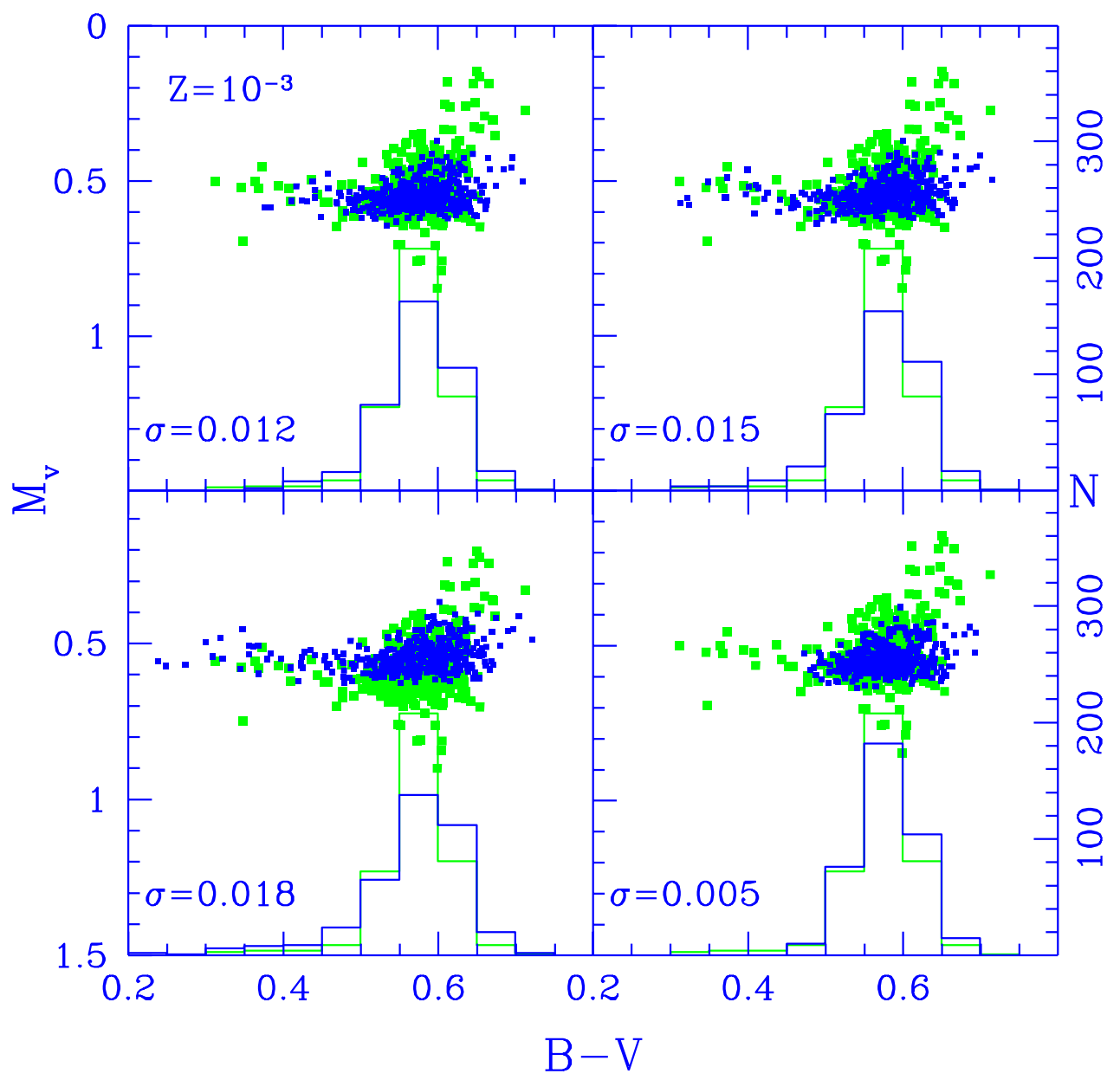

Fig. 4. - Simulations for the red clump of the HB of NGC 2808. The assumed age is 13Gyr. The gaussian spread $\sigma$ added to the average mass loss of $0.13 \mathrm{M}_{\odot}$ along the RGB is indicated in the panels. The simulations are compared with the distribution of the clump stars. The distance modulus and reddening are $(\mathrm{m}-\mathrm{M})_{0}=15.25$ and $\mathrm{E}(\mathrm{B}-\mathrm{V})=0.18$. The ordinates on the right give the number of stars in the histograms. Mass spreads from $0.012 \mathrm{M}_{\odot}$ to $0.018 \mathrm{M}_{\odot}$ are consistent with the observed lack of stars in the RR Lyrae gap. 


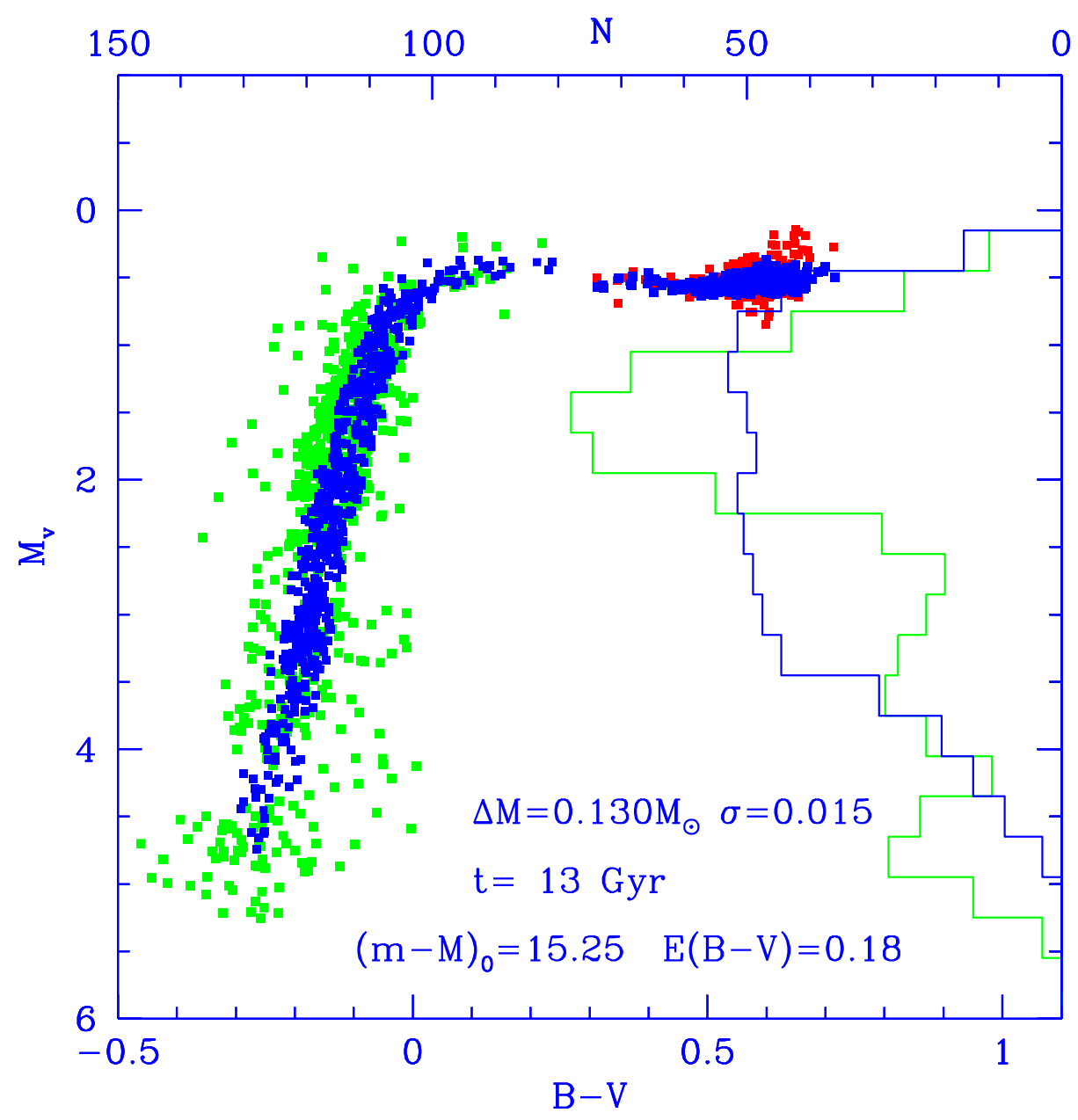

Fig. 5.- Typical simulation obtained assuming an age of $13 \mathrm{Gyr}$. The average mass loss on the RGB has been assumed $0.13 \mathrm{M}_{\odot}$ in order to reproduce the red clump. The corresponding blue HB distribution, assuming random helium content between 0.27 and 0.32 is shown. Notice that the difference in magnitude between the red clump and the red side of the blue HB ( this latter being about 0.08mag more luminous) is consistent with the increase in the HB luminosity due to the helium difference between the two sides. 

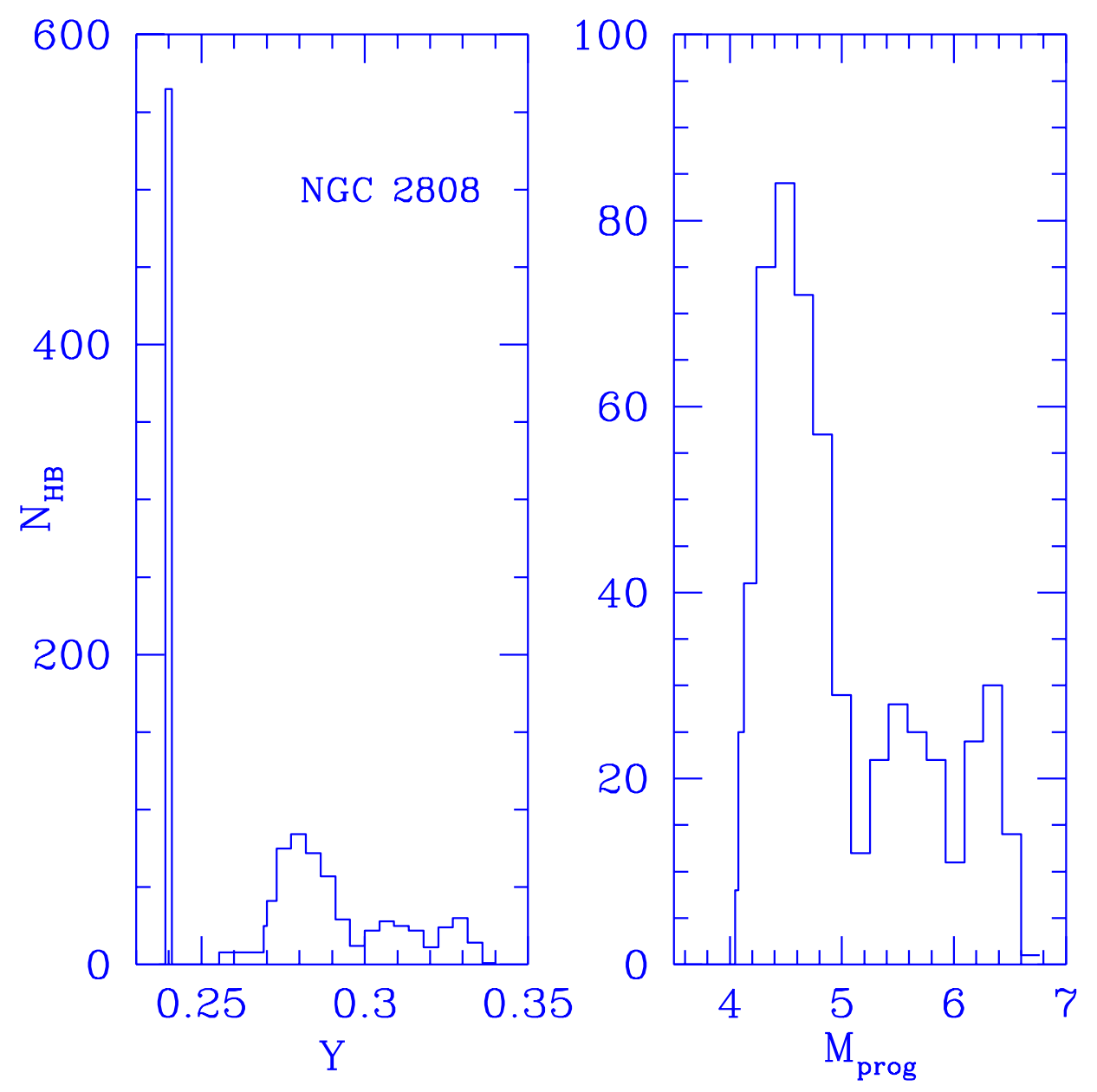

Fig. 6.- Number of stars versus helium abundance (left) and corresponding range of initial mass progenitor (right) in the HB of NGC 2808. The assumptions are: 1) that the red clump comes from a population with the initial helium abundance $\mathrm{Y}=0.24 ; 2)$ that the location of stars along the blue side of the HB is due to the same process of mass loss (average mass loss and spread) which is consistent with the red clump and an age of $13 \mathrm{Gyr}$, but the total mass is also a function of the initial helium content of the stars, according to equation 1 ; 3) that the material forming the blue HB stars has the helium content of the progenitor AGB stars, according to the relation 2. 


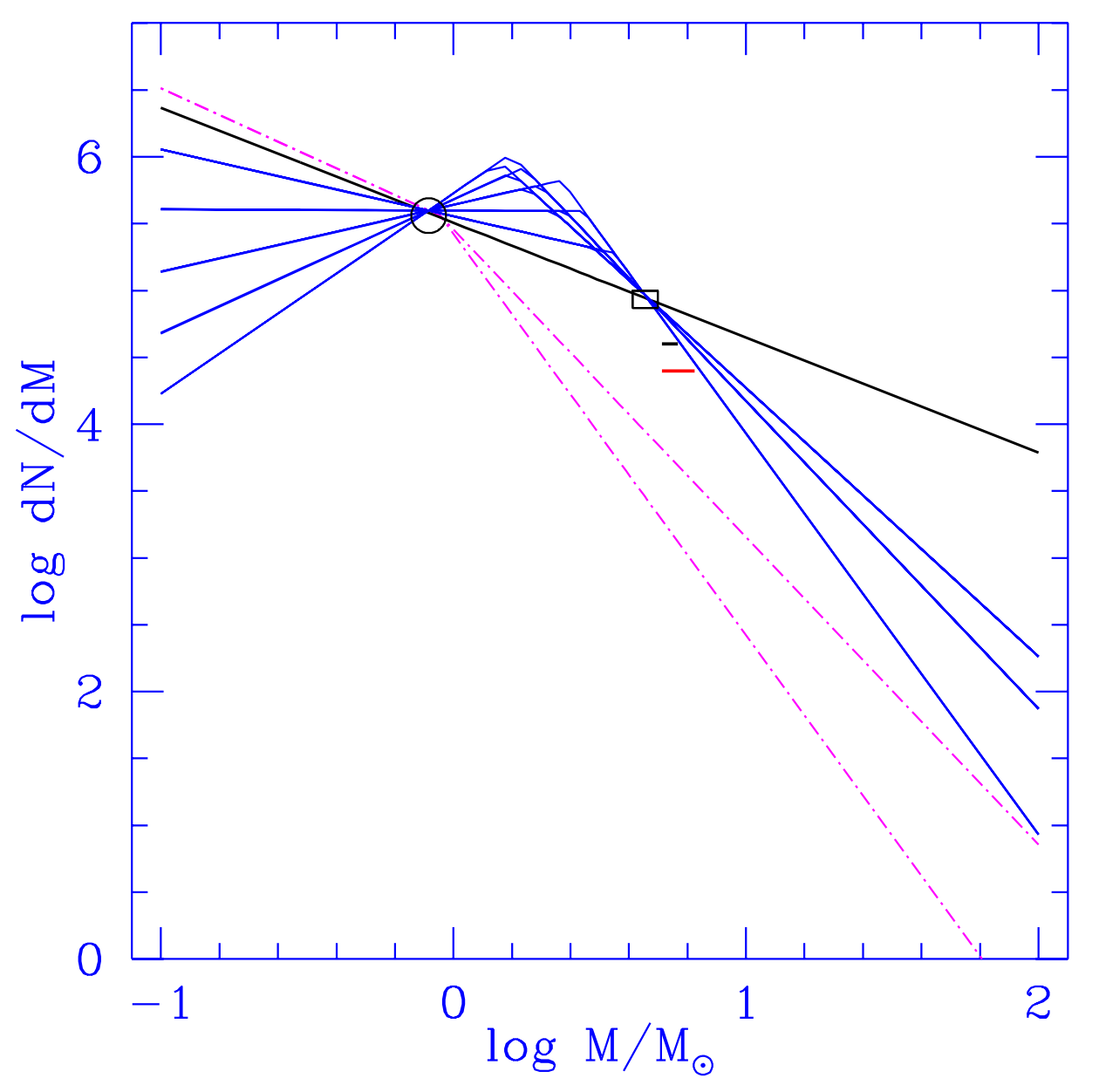

Fig. 7.- Possible choices for the IMFs of the first generation stars in NGC 2808, based on the constraints of the HB morphology. The circle at $\mathrm{M} \simeq 0.82 \mathrm{M}_{\odot}$ refers to the number of the red clump stars, $N_{\text {clump }}=$ 565 from Bedin et al. (2000). The square at $\mathrm{M} \simeq 4-5 \mathrm{M}_{\odot}$ refers to the number of stars in the EBT1 $\left(N_{B H B}=401\right)$. The continuous straight line passing through the circle and the rectangle is a unique mass function of slope $\mathrm{x}=-0.14$ (see Table 1 ). The two dash-dotted lines are IMFs built with $\mathrm{x}=0$ at $\mathrm{M}<0.9 \mathrm{M}_{\odot}$, and $\mathrm{x}=1.3$ or $\mathrm{x}=2$ at $\mathrm{M}>0.9 \mathrm{M}_{\odot}$, following the experimental determination of the IMF in the Galaxy (Kroupa 2001). At $\mathrm{M}=5.1-6 \mathrm{M}_{\odot}$ and $\mathrm{M}=5.1-6.6 \mathrm{M}_{\odot}$ we show the IMF points based on the number of stars in EBT2 and EBT3 taken together (see text). 